\title{
Multi-resonant optical parametric oscillator without mirrors based on 1D and 2D-PPLT nonlinear photonic crystal
}

\author{
Safia Mohand Ousa id ${ }^{1}$, Mahmoud Chakaroun ${ }^{1}$, Kai-hsun Chang ${ }^{2}$, Thierry Billeton ${ }^{1}$, L-Han Peng ${ }^{2}$, and Azzedine Boudrioua ${ }^{1}$ \\ 1. Laboratoire de Physique de Lasers CNRS UMR 7538, Université Paris 13, Sorbonne Paris Cité, Villetaneuse, France \\ 2. Graduate Institute of Photonics and Optoelectronics, GIPO, National Taiwan University, Taipei, 106, Taiwan
}

Abstract: In this work, we demonstrate that it is possible to obtain an optical parametric oscillator with a simultaneous amplification of multiple frequencies using only a nonlinear photonic crystal with highly polished end-faces, preventing the utilization of external cavity mirrors.

\section{Introduction}

Optical parametric oscillator (OPO) is an efficient tool to provide powerful parametric sources. It represents a very promising device for various technological applications. OPO is a laser oscillator where the ga in depends on phase matching conditions, which link the pump wave $\left(\omega_{\mathrm{p}}\right)$ and the two waves generated (signal $\omega_{\mathrm{s}}$ and the complementary wave or idler $\left.\omega_{\mathrm{i}}\right)$ by the second order nonlinear susceptibility $\chi^{(2)}$ of the gain material. Conventional OPO consists of placing a $\chi^{(2)}$ non-linear medium in an optical cavity resonator. Most of OPOs are single resonant at either signal or idler wavelengths. Many other studies were interested in the cavity design in order to achieve a double and triple resonant OPO. Since its first demonstration in 1995 by Meyer et al. [1], several works have been carriedout to improve OPO efficiency and stability by optimizing the cavity mirrors. However, the use of a Fabry-Perot cavity-type with a specific design of the mirrors makes the device cumbersome and add more complexity to manage all the resonances together.

In this work, we report the demonstration of optical parametric oscillation using only a nonlinear photonic crystal polished in a particular way. The highly polished end-faces of the sample act as cavity mirrors, which prevent the utilization of external mirrors. Moreover, we show that it is possible to obtain, simultaneously, an optical parametric oscillation of multiple frequencies due to cascaded nonlinear interactions.

\section{Results and discussion}

The demonstration of multi-resonant optical oscillation without mirror in nonlinear photonic crystals of lithium tantalate is almost fortuitous. It appeared during a comparison study of multi-wavelength generation by cascade effect in photonic crystals $1 \mathrm{D}$ and $2 \mathrm{D}$-PPLT. In order to improve the efficiency of this nonlinear process, we developed a specific polishing technique to obta in a very high optical quality of the sample faces. It includes lapping, softening, fast flat polishing and a final polishing to obtain a precise quality of flatness and roughness of the surface. The samples used are $\mathrm{LiTaO}_{3}$ nonlinear photonic crystal of $20 \times 10 \times 0.5 \mathrm{~mm}^{3}$ periodically polarized by electric poling in one dimension (1D-PPLT) and two dimensions (2D-PPLT) with a period $\Lambda=8.52 \mu \mathrm{m}$. The $2 \mathrm{D}$-PPL T is a square lattice with a circular pattern and a filling factor of
$38 \%$. The one in $1 \mathrm{D}$ is $50 \%$. The filling factors are chosen to obtain the most efficient frequency conversion for these two structures [2]. For the characterization, we used the same experimental setup as depicted in reference [3]. The measurements are carried at $110^{\circ} \mathrm{C}$ which corresponds to the optimum conversion efficiency temperature. In these conditions, the measured out-put spectrum presents unexpected additional wavelengths as displayed in figure 1.a. (5 new peaks are observed).
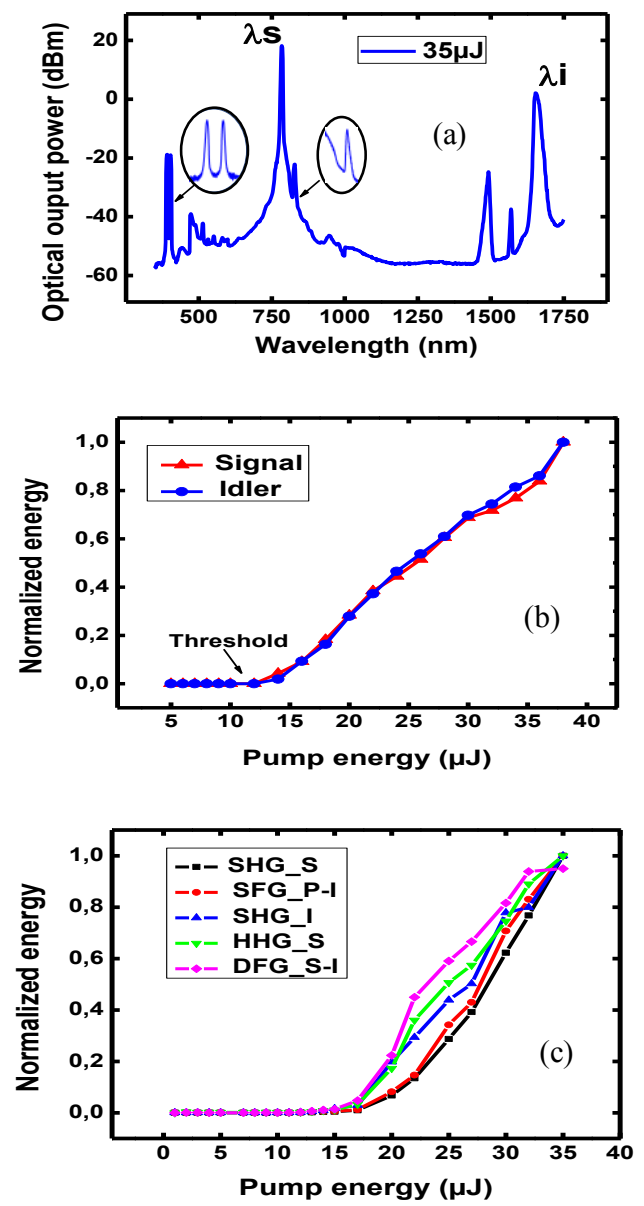

Fig.1. (a) The generated multi-wavelengths spectrum. (b) Threshold measurements of signal an idler and (c) of cascaded nonlinear processes. From 1D-PPLT nonlinear photonic crystal at $\mathrm{T}=110^{\circ} \mathrm{C}$. 
As already stated in references [3, 4], it was found that the interaction of the signal $\lambda_{\mathrm{s}}$ at $787 \mathrm{~nm}$, the idler $\lambda_{\mathrm{i}}$ at $1641 \mathrm{~nm}$ (in Fig.1a), and the pump can further lead to several cascaded nonlinear processes: SHG of the signal at $394 \mathrm{~nm}$, SHG of the idler at $820 \mathrm{~nm}$, SFG of the pump and the idler at $402 \mathrm{~nm}$, DFG of the signal and the idler at $1516 \mathrm{~nm}$ and the half $\mathrm{HG}$ of the signal $1576 \mathrm{~nm}$. However, the phase matching conditions, alone, cannot explain the occurrence of these processes effectively. In order to explain the physical origin of this phenomenon, we suggest considering resonant oscillations inside the sample, which in turn sustain the cascaded nonlinear process. To confirm this assumption, we measured the energy threshold for each peak. Figures 1(b) and 1(c) show the obtained results for signal, idler and the other cascaded processes for the $1 \mathrm{D}$ crystal. It is clearly seen that these nonlinear processes are subject to a threshold phenomenon, which emphasize a resonant behaviour. In addition to that, we studied the spectrum variation of the obtained peaks at different energy pumps before and after the threshold.
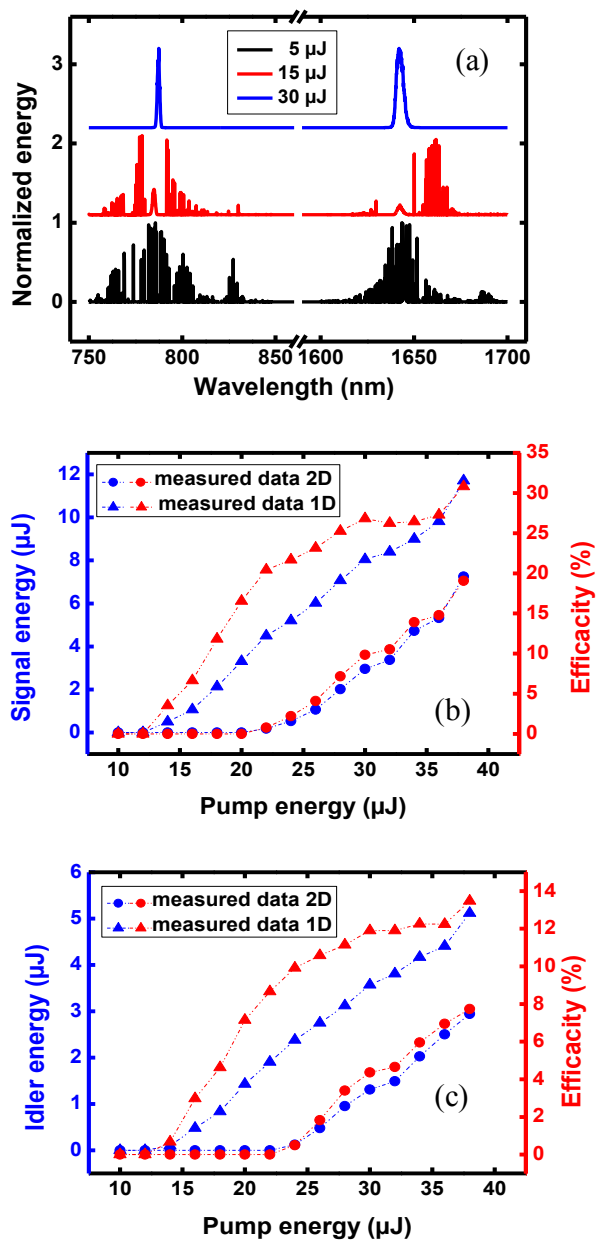

Fig.2. (a) The broad spectrum of signal and idler waves generated from a 1D-PPLT nonlinear photonic crystal before the threshold $\left(E_{\mathrm{p}}=5 \mathrm{~J}\right)$, near and at three time of the threshold. The conversion efficiency of 1D and 2D-PPLT at $110^{\circ} \mathrm{C}$ (b) Signal (787nm), (c) Idler (1641nm).
The signal and idler oscillation threshold is estimated at $12 \mathrm{~J}$ for $1 \mathrm{D}-\mathrm{PPLT}$ and $20 \mathrm{~J}$ for 2D-PPLT, that of the other waves generated by cascade effect is reached after an increase of the pump energy of $4 \mathrm{~J}$. The oscillation threshold of 2D-PPLT is relatively high compared to the 1D-PPLT, due to its low conversion efficiency. The frequencies obtained by cascade effects require a fairly powerful signal and idler and therefore greater pump energy.

As an example, the figures 2.a display the signal and the idler spectrum recorded for the 1D-PPLT before, near and at three times the threshold $\left(E_{\mathrm{p}}=10 \mathrm{~J}\right)$. Before the threshold, the spectrum consists of several peaks due to the multi-wavelength nature of the OPG process in the nonlinear photonic crystal PPLT. Just above the threshold, the number of peaks decrease and there two peaks at $787 \mathrm{~nm}$ and $1641 \mathrm{~nm}$ of low intensity appear. For a pump power equal to 3 times the oscillation threshold, we see that the 2 peaks are increased. This behavior is explained by the competition between different coupled interactions inside the nonlinear photonic crystal; only the two powerful peaks corresponding to the couple $\omega_{\mathrm{s}}$ and $\omega_{\mathrm{i}}$ which minimize the phase mismatch remain. These results emphasize that the cavity formed by the sample faces acts as a filter to select the most effective interaction. Note that all the previously observed peaks behave in the same way, confirming the oscillation phenomenon.

Eventually, the maximum total conversion efficiency achieved is $45 \%$ for $1 \mathrm{D}-\mathrm{PPLT}$ and $27 \%$ for $2 \mathrm{D}-\mathrm{PPLT}$. That of the signal $(787 \mathrm{~nm})$ is $30 \%$ for $1 \mathrm{D}$-PPLT, while it is $18 \%$ for the 2D-PPLT. For the idler (1641 nm), the maximum efficiency obtained in the PPLT-1D is $15 \%$ and it is $9 \%$ in the PPLT-2D. The experimental measurements obtained show that the efficiency of 1D-PPLT is 1.7 times greater than that of 2D-PPLT, which is in good agreement with the results reported in the literature [3].

\section{Conclusion}

In this work, we report evidence of optical parametric oscillations in 1D and 2D-PPL T nonlinear photonic crystal without using external mirrors. The oscillation threshold and the efficiency of the device should be greatly improved by using crystals with antireflection-treated sides.

\section{References}

[1] L. Myers, R. Eckardt, M. Fejer, R. Byer, W. Bosenberg, and J. Pierce. "Quasi phase matched optical parametric oscillators in bulk periodically poled $\mathrm{LiNbO}_{3}$," JOSA B, 12, p. 2102,(1995).

[2] Broderick, N. G. R., Ross, G. W., Offerhaus, H. L., Richards on, D. J., \& Hanna, "Hexagonally Poled Lithium Niobate: A Two-Dimensional Nonlinear Photonic Crystal'. PRL, 84(19), 4345-4348, (2000).

[3] S. Mohand Ousaid, J-Y. Han, K-H. Chang, M. W. Lee, M. Chakaroun, L.-H. Peng and A. Boudrioua, "Magic crossing points of temperature dependent cascaded frequency conversions in nonlinear photonic crystals', CLEO Munich (2019).

[4] Zohra Yellas, Min Won Lee, Régis Kremer, Kai-Hsun Chang, Mahmoud R. Beghoul, Lung-Han Peng, and Azzedine Boudrioua "Simultaneous frequency conversions in nonlinear photonic crystals of lithium tantalitewith a square lattice", CLEO Munich (2017) 